Article

\title{
Bifurcation Phenomena Studies of a Voltage Controlled Buck-Inverter Cascade System
}

\author{
Xiaofei $\mathrm{Li}^{1}{ }^{1}$, Chunsen Tang ${ }^{1, *}$, Xin Dai ${ }^{1}$, Aiguo Patrick $\mathrm{Hu}^{2}$ and Sing Kiong Nguang ${ }^{2}$ \\ 1 School of Automation, Chongqing University, Chongqing 400044, China; xiaofea_lee@163.com (X.L.); \\ toybear@vip.sina.com (X.D.) \\ 2 Department of Electrical and Computer Engineering, The University of Auckland, 1142 Auckland, \\ New Zealand; a.hu@auckland.ac.nz (A.P.H.); sk.nguang@auckland.ac.nz (S.K.N.) \\ * Correspondence: cstang@cqu.edu.cn; Tel.: +86-23-6511-2570
}

Academic Editor: Gabriele Grandi

Received: 5 April 2017; Accepted: 14 May 2017; Published: 18 May 2017

\begin{abstract}
This paper studies the complex bifurcation phenomena of a voltage-controlled Buck-inverter cascade system. A state-flow chart is drawn to illustrate the complex relations among the linear operating modes. Combined with the state transition function of each mode, the time response of the system can be obtained. For period-one steady state, the periodic mapping function and its fixed point are further derived, on the basis of which the Jacobi matrix is developed and its maximum eigenvalue is analyzed to understand the bifurcation diagram. By globally analyzing the state space using this cell mapping method, the coexistence of attractors is revealed in the Buck-inverter system. All theoretical results have been verified experimentally on a prototype system. The results obtained can be used for guiding the design and analysis of the Buck-inverter system. The analyzing method can be helpful for studying other power electronics systems with compound topologies.
\end{abstract}

Keywords: Buck-inverter; bifurcation; Jacobi matrix; coexistence of attractors; cell mapping

\section{Introduction}

Buck-inverter cascade configuration is widely used in wireless power transfer (WPT) and inductive heating applications for high frequency magnetic field generation [1-3]. Usually, the inverter is used to drive a resonant network to generate high-frequency alternating current along the transmitter coil [2] or the heating coil [4], and the Buck converter is used to regulate the output power. However, due to the interactions between the Buck converter and the load resonant inverter, and the nonlinear switching effect of the switches, nonlinear characteristics are inevitable issues to consider when designing a Buck-inverter system [5].

In fact, nonlinear characteristics are common in power electronics systems. Various nonlinear phenomena in power electronics have been found in the past two decades. The initial research focused on DC/DC unit converters. Period-doubling bifurcation and chaos were found in Buck, Boost, Buck-Boost and Sepic converters, etc. [6-12]. Particularly, the coexistence of the attractor's phenomenon was found in Buck converters. Starting from different initial values, the converters converged to different steady states [13,14]. Flip bifurcation and chaos were found in a three-state boost switching regulators, and the results show the system exhibited a typical route to chaos via period-doubling [15]. Later, nonlinear phenomena in AC/DC converters were studied. Reference [16] studied the fast-scale instability in a power-factor-correction (PFC) Boost converter, and bifurcation near the switching moment was observed. Nonlinear phenomena in DC/AC inverters were first studied in [17], and border collision bifurcation occurred when varying the control parameters. Furthermore, Hopf bifurcation was also observed in DC/AC inverters, and it has been verified that Hopf bifurcation is also a route to chaos [18]. Reference [19] studied chaos and the coexistence of attractors' phenomena 
in a full-bridge converter. The results show that the period-one attractor co-exists with the period-three attractor at some critical parameter values. Moreover, there are also some studies of nonlinear behaviors in high-order converters [20-22]. Bifurcation and chaos were found in parallel Buck or Boost converters. Unlike parallel converters, the authors of $[23,24]$ studied the nonlinear characteristics of a load resonant $\mathrm{DC} / \mathrm{DC}$ combined converter, which is a cascade system with a DC/AC inverter series connected to an AC/DC converter, and a symmetry-breaking bifurcation was introduced in this research. All of the above studies showed that complex nonlinear phenomena can exist in various power electronics converters because of the switching transitions among different circuit topologies [24,25]. Studies on the nonlinear behaviors help us to better understand the operation principles of the power electronics systems and can furthermore lead to innovative techniques in dealing with the nonlinearities.

When analyzing the nonlinear phenomena of the converters, a discrete mapping modeling method is commonly used. The method can quickly determine the periodic state, so as to plot bifurcation diagrams [6,11,12]. For combined parallel and series converters, the nonlinear phenomena are much more complex than in single unit converters, which have been proven to be more difficult in being modeled and analyzed [26,27]. In this paper, to better analyze the operating principle of the Buck-inverter cascade system, a state-flow chart has been introduced to illustrate the complex relations among the linear operating modes. In order to globally analyze the coexistence of attractors, the cell mapping method is also introduced. Utilizing these methods, bifurcation diagrams with the reference voltage and the input voltage as bifurcation parameters are studied and verified by the maximum Jacobi matrix eigenvalues. In particular, the coexistence of attractors is observed theoretically and verified experimentally.

The paper is structured as follows: the operating principle, state-flow chart and Jacobi matrix analysis of the Buck-inverter system is derived in Section 2; bifurcation diagrams and theoretical verification by the maximum Jacobi matrix eigenvalues are shown in Section 3; the coexistence of attractors are revealed and investigated globally with the cell mapping analytical method in Section 4; the experiment verification of the bifurcation diagrams and coexistence of attractors are presented in Section 5; and finally, the conclusions are drawn in Section 6.

\section{Modeling of Voltage-Controlled Buck-Inverter}

\subsection{Operating Principles of Voltage-Controlled Buck-Inverter}

The diagram of the voltage-controlled Buck-inverter cascade system is shown in Figure 1. The main topology consists of two parts. One is a Buck converter and the other is a full-bridge load resonant inverter. A voltage closed loop is added to the output voltage of the Buck converter to adjust the output power of the system. $E$ is the input DC source. $S$ and $D$ are the switch and the fly-wheeling diode of the Buck circuit, respectively. $L$ and $C$ constitute a low pass filter. $R_{L}$ is the equivalent series resistance of $L . S 1, S 2, S 3$ and $S 4$ constitute a full-bridge inverter. $L_{p}$ and $C_{p}$ constitute a series resonant circuit to obtain a high frequency sinusoidal alternating current in the coil $L_{p} . R_{p}$ is the equivalent load. To achieve a soft switching operating mode, switches $S 1-S 4$ should commutate at the instance when the current $i_{p}$ crosses zero [28,29]. In the voltage feedback control loop, $u_{r e f}$ is the reference voltage and $u_{c}$ is the output voltage of the Buck converter which is sensed by a voltage divider with a ratio of $k$ and subtracted from the reference voltage $u_{r e f} a$ is the gain of the error amplifier which can also be regarded as a proportional controller.

The control voltage $u_{c o n}$ shown in Figure 1 can be written as:

$$
u_{c o n}=a\left(u_{r e f}-k u_{c}\right)
$$

The saw-tooth carrier signal in one control period is given as:

$$
u_{\text {ramp }}=U_{L}+\left(U_{H}-U_{L}\right) t, t \in\left[0, T_{b}\right]
$$


where $t$ is the time variable; $T_{b}\left(T_{b}=1 / f_{b}, f_{b}\right.$ is the frequency of the Buck converter) is the period of the saw-tooth signal; $U_{L}$ and $U_{H}$ are its lower and upper threshold values respectively.

The negative voltage feedback control strategy of the Buck circuit is:

$$
u_{g}= \begin{cases}1 & u_{c o n} \geq u_{\text {ramp }} \\ 0 & u_{c o n}<u_{\text {ramp }}\end{cases}
$$

To achieve soft switching, a fixed frequency which is consistent with the natural frequency of the resonant network is used to control the inverter. The control sequence in one period can be written as:

$$
\left\{\begin{array}{l}
u_{14}=1, u_{23}=0, t \in\left[0, \frac{T_{i}}{2}\right] \\
u_{23}=1, u_{14}=0, t \in\left(\frac{T_{i}}{2}, T_{i}\right]
\end{array}\right.
$$

where $t$ is the time variable, and $T_{i}\left(T_{i}=1 / f_{i}, f_{i}\right.$ is the frequency of the resonant inverter) is the control period of the resonant inverter.

\subsection{State-Flow Chart of Buck-Inverter System}

Usually, when analyzing power electronics systems, we divide the system into a series of operation modes according to the on/off state of the controlled and non-controlled switches and study the modes one by one [30,31]. Accordingly, the Buck-inverter system shown in Figure 1 can be piecewise linearized into six possible working modes because the Buck converter works in continuous current mode (CCM) and discontinuous current mode (DCM):

(1) Mode 1: $S, S 1, S 4$ on and $D, S 2, S 3$ off;

(2) Mode 2: $D, S 1, S 4$ on and $S, S 2, S 3$ off;

(3) Mode 3: $S, S 2, S 3$ on and $D, S 1, S 4$ off;

(4) Mode 4: D, S2, S3 on and $S, S 1, S 4$ off;

(5) Mode 5: $S 1, S 4$ on and $S, D, S 2, S 3$ off;

(6) Mode 6: $S 2, S 3$ on and $S, D, S 1, S 4$ off.

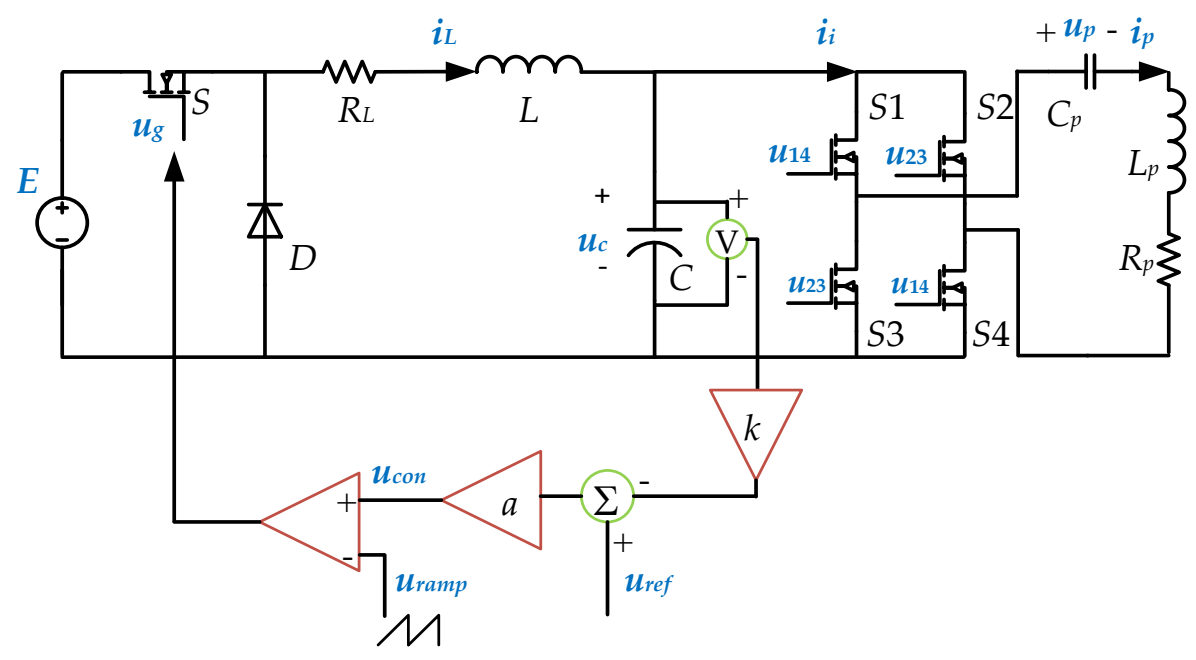

Figure 1. Diagram of a voltage-controlled Buck-inverter cascade system.

The equivalent circuits of these six working modes are shown in Figure 2a-f respectively.

To reveal the complex relations among the working modes, the state-flow chart of the six working modes is drawn and shown in Figure 3, where $t_{0}$ is the time when $i_{L}$ falls to zero when the Buck 
converter works in DCM. $d$ is the duty cycle of Buck converter. The system starts from Mode 1 . The direction of the arrows indicates the flow of the working modes, while the texts on the arrows indicate the mode switching conditions. Black lines indicate the flow cases when only considering the $\mathrm{CCM}$ of the Buck converter, while red lines indicate the further flow cases when considering the DCM of the Buck converter.

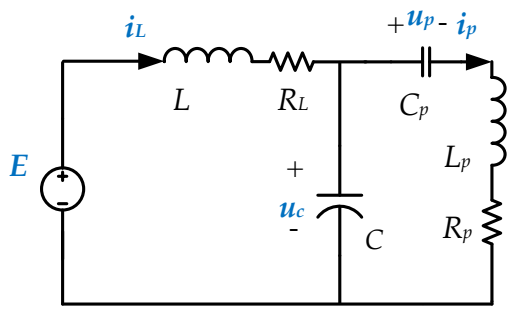

(a)

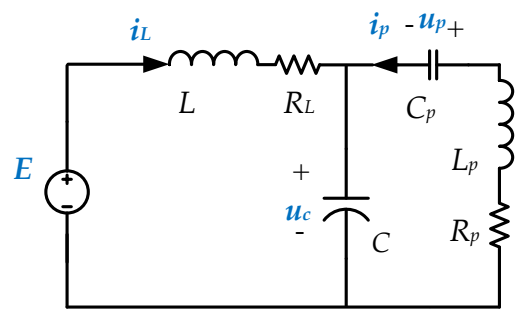

(c)

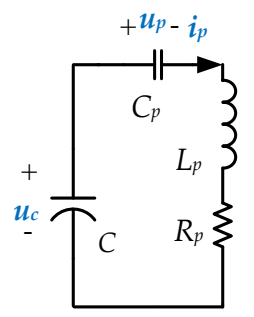

(e)

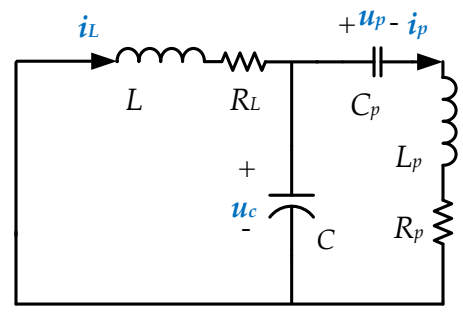

(b)

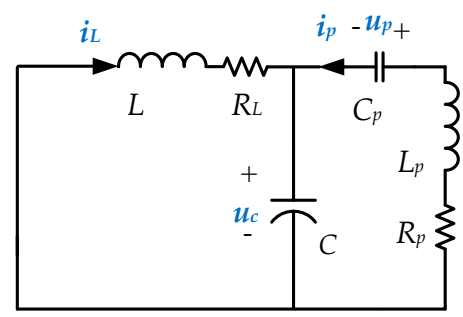

(d)

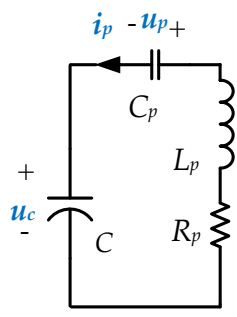

(f)

Figure 2. Equivalent circuits of the six working modes of the Buck-inverter system: (a) Mode 1; (b) Mode 2; (c) Mode 3; (d) Mode 4; (e) Mode 5; (f) Mode 6.

Choose $X=\left[\begin{array}{llll}i_{L} & u_{c} & i_{p} & u_{p}\end{array}\right]^{T}$ and $U=[E]$ as the state vector and the input vector of the Buck-inverter respectively. Then the state space model of these six working modes can be derived according to Kirchhoff's law:

$$
\left\{\begin{array}{cc}
\dot{X}=A_{1} X+B_{1} U, & \text { Mode1 } \\
\dot{X}=A_{2} X+B_{2} U, & \text { Mode2 } \\
\dot{X}=A_{3} X+B_{3} U, & \text { Mode3 } \\
\dot{X}=A_{4} X+B_{4} U, & \text { Mode4 } \\
\dot{X}=A_{5} X+B_{5} U, & \text { Mode5 } \\
\dot{X}=A_{6} X+B_{6} U, & \text { Mode6 }
\end{array}\right.
$$

where $A_{1}=A_{2}=\left[\begin{array}{cccc}\frac{-R_{L}}{L} & \frac{-1}{L} & 0 & 0 \\ \frac{1}{C} & 0 & \frac{-1}{C} & 0 \\ 0 & \frac{1}{L_{P}} & \frac{-R_{p}}{L_{P}} & \frac{-1}{L_{P}} \\ 0 & 0 & \frac{1}{C_{P}} & 0\end{array}\right], A_{3}=A_{4}=\left[\begin{array}{cccc}\frac{-R_{L}}{L} & \frac{-1}{L} & 0 & 0 \\ \frac{1}{C} & 0 & \frac{1}{C} & 0 \\ 0 & \frac{-1}{L_{P}} & \frac{-R_{p}}{L_{P}} & \frac{-1}{L_{P}} \\ 0 & 0 & \frac{1}{C_{P}} & 0\end{array}\right]$, $A_{5}=\left[\begin{array}{cccc}0 & 0 & 0 & 0 \\ 0 & 0 & \frac{-1}{C} & 0 \\ 0 & \frac{1}{L_{P}} & \frac{-R_{p}}{L_{P}} & \frac{-1}{L_{P}} \\ 0 & 0 & \frac{1}{C_{P}} & 0\end{array}\right], A_{6}=\left[\begin{array}{cccc}0 & 0 & 0 & 0 \\ 0 & 0 & \frac{1}{C} & 0 \\ 0 & \frac{-1}{L_{P}} & \frac{-R_{p}}{L_{P}} & \frac{-1}{L_{P}} \\ 0 & 0 & \frac{1}{C_{P}} & 0\end{array}\right], B_{1}=B_{3}=\left[\begin{array}{c}1 \\ L \\ 0 \\ 0 \\ 0\end{array}\right], B_{2}=B_{4}=B_{5}=B_{6}=\left[\begin{array}{c}0 \\ 0 \\ 0 \\ 0\end{array}\right]$. 
The analytical solution of each linearized mode can be described as follows according to [32]

$$
X(t)= \begin{cases}\Phi_{1}(t) X_{10}+\left(\Phi_{1}(t)-I\right) A_{1}{ }^{-1} B_{1} E & \text { Mode 1 } \\ \Phi_{2}(t) X_{20} & \text { Mode 2 } \\ \Phi_{3}(t) X_{30}+\left(\Phi_{3}(t)-I\right) A_{3}{ }^{-1} B_{3} E & \text { Mode 3 } \\ \Phi_{4}(t) X_{40} & \text { Mode 4 } \\ \Phi_{5}(t) X_{50} & \text { Mode 5 } \\ \Phi_{6}(t) X_{60} & \text { Mode 6 }\end{cases}
$$

where $i(i=1,2,3,4,5,6)$ is the mode number and $\Phi_{i}(t)=e^{A i t}, X_{i 0}$ is the initial states of each working mode.

According to the mode transition chart shown in Figure 3 and the state transition function of each working mode shown in Equation (6), the dynamical time responses of the Buck-inverter system can be derived for any given initial states with the strategies of the Buck converter and the resonant inverter as shown in Equations (3) and (4).

As discussed in [32], if the steady state mode series are known, the steady state responses are able to be predicted analytically. However, for the Buck-inverter cascade system, if $T_{b}$ is different from $T_{i}$, the steady state mode series will be unknown especially when $T_{b}$ and $T_{i}$ are relatively prime. In this case, the steady state periodic mapping function cannot be derived and neither can the Jacobi matrix. The nonlinearity of the system can only be studied through numerical simulations. In order to better understand the bifurcation characteristics of the system, we assume $T_{b}=T_{i}$ and the phase difference is zero in the following sections.

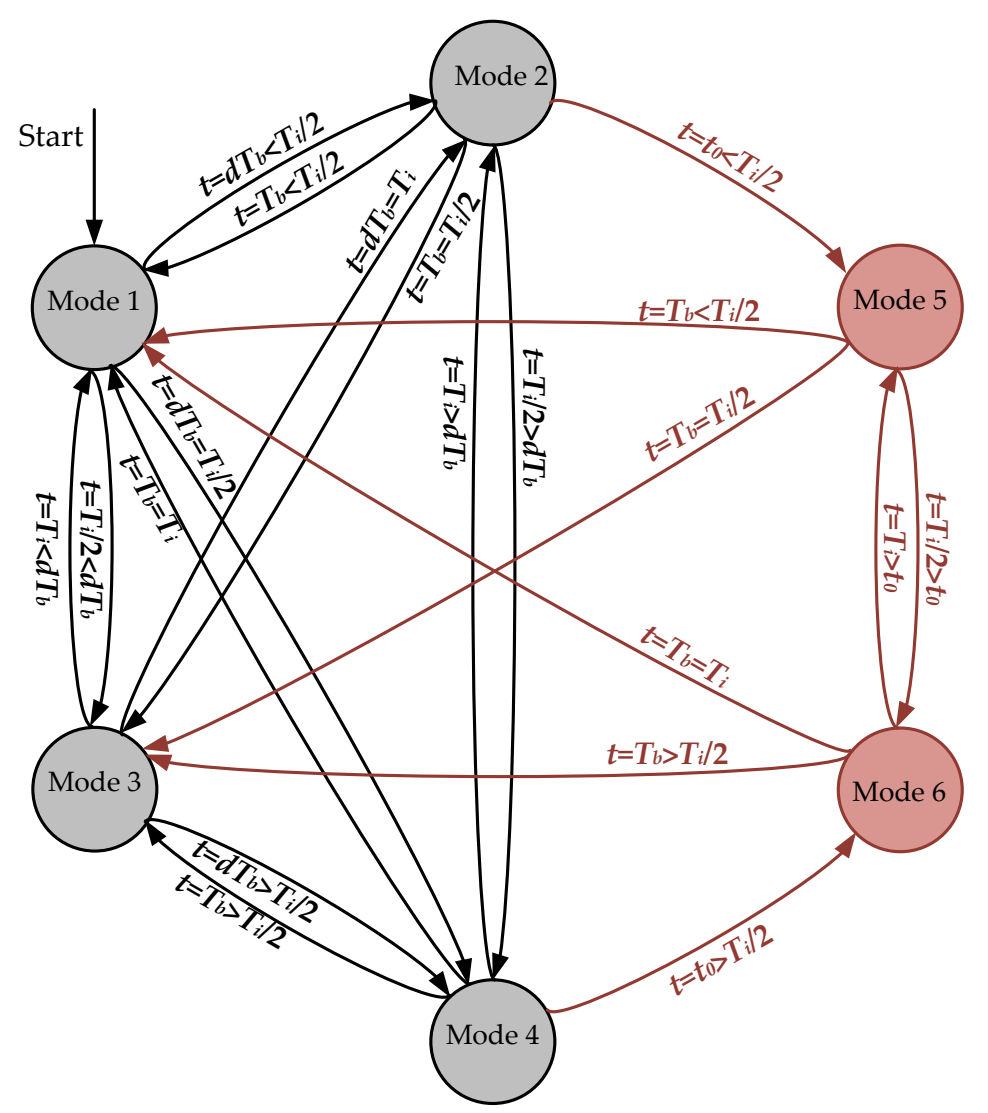

Figure 3. State-flow chart of Buck-inverter system. 


\subsection{Stability Analysis of Period-One Steady State}

The maximum Jacobi matrix eigenvalue is an effective way to verify the stability of the system at period-one steady state [33,34]. If the absolute value of the maximum Jacobi matrix eigenvalue is larger than 1, the period-one operating point is unstable (there is bifurcation or chaos).

To simplify, assume that the Buck converter works in CCM, since the analysis process is the same when Buck works in DCM. When the Buck converter goes into period-one steady state, the state flow is different from when the duty cycle $d$ of the Buck converter is larger or smaller than 0.5. When $d$ is larger than 0.5 , the state flow in the steady period is: Mode 1, Mode 3, Mode 4. And the time spans of the three modes are $\xi_{1}=T_{b} / 2, \xi_{3}=d T_{b}-T_{b} / 2$ and $\xi_{4}=T_{b}-d T_{b}$ respectively. When time variable $t$ is smaller than $T_{b} / 2$, the Buck-inverter works in Mode 1 , and then it goes into Mode 3 when $t=T_{b} / 2$. Finally, the system goes into Mode 4 when $t=d T_{b}$. Otherwise, when $d$ is smaller than 0.5 , the state-flow in the steady period is: Mode 1, Mode 2, Mode 4 . And the time spans of the three modes are $\xi_{1}=d T_{b}, \xi_{2}=T_{b} / 2-d T_{b}$ and $\xi_{4}=T_{b} / 2$ respectively. When the time variable $t$ is smaller than $d T_{b}$, the Buck-inverter works in Mode 1, and then goes into Mode 2 when $t=d T_{b}$. Finally, the system goes into Mode 4 when $t=T_{b} / 2$.

The analytical solution of each linearized mode in one period can be described as follows when $d$ is larger or smaller than 0.5 .

$$
\begin{aligned}
d \geq 0.5: & X(t)= \begin{cases}\Phi_{1}(t) X_{0}+\left(\Phi_{1}(t)-I\right) A_{1}{ }^{-1} B_{1} E & t \in\left[0, \frac{T_{b}}{2}\right] \\
\Phi_{3}\left(t-\frac{T_{b}}{2}\right) X\left(\frac{T_{b}}{2}\right)+\left(\Phi_{3}\left(t-\frac{T_{b}}{2}\right)-I\right) A_{3}{ }^{-1} B_{3} E & t \in\left(\frac{T_{b}}{2}, d T_{b}\right] \\
\Phi_{4}\left(t-d T_{b}\right) X\left(d T_{b}\right) & t \in\left(d T_{b}, T_{b}\right]\end{cases} \\
d<0.5: & X(t)= \begin{cases}\Phi_{1}(t) X_{0}+\left(\Phi_{1}(t)-I\right) A_{1}{ }^{-1} B_{1} E & t \in\left[0, d T_{b}\right] \\
\Phi_{2}\left(t-d T_{b}\right) X\left(d T_{b}\right) & t \in\left(d T_{b}, \frac{T_{b}}{2}\right] \\
\Phi_{4}\left(t-\frac{T_{b}}{2}\right) X\left(\frac{T_{b}}{2}\right) & t \in\left(\frac{T_{b}}{2}, T_{b}\right]\end{cases}
\end{aligned}
$$

where $i(i=1,2,3,4)$ is the mode number, $X_{0}=\left.X(t)\right|_{t=0}$ and $\Phi_{i}(t)=e^{A i t}$.

Equation (7) results in six discrete mapping functions:

$$
f_{i, \zeta_{i}}(X)=\Phi_{i}\left(\xi_{i}\right) X+A_{i}^{-1}\left(\Phi_{i}\left(\xi_{i}\right)-I\right) B_{i} U \quad i=1,2,3,4 .
$$

Let $X_{0}$ be the initial state and $n=0,1,2, \ldots$ be the index of the discrete map. $X_{n}$ and $X_{n+1}$ are the initial state and final state of each operating period, respectively. The duty cycle between the $n$ to $n+1$ interval is $d_{n}$, then $\xi_{1}=T_{b} / 2, \xi_{3}=d_{n} T_{b}-T_{b} / 2$ and $\xi_{4}=T_{b}-d_{n} T_{b}$ when $d$ is larger than 0.5 while $\xi_{1}=d_{n} T_{b}, \xi_{2}=T_{b} / 2-d_{n} T_{b}$ and $\xi_{4}=T_{b}-T_{b} / 2$ when $d$ is smaller than 0.5 . Based on (8), the stroboscopic mapping model $F$ can be obtained as:

$$
\begin{aligned}
d \geq 0.5: \quad X_{n+1} & =F\left(X_{n}, \xi_{1}, \xi_{3}, \xi_{4}\right)=f_{4, \xi_{4}} \circ f_{3, \xi_{3}} \circ f_{1, \xi_{1}}\left(X_{n}\right) \\
& =\Phi_{4}\left(\xi_{4}\right)\left(\Phi_{3}\left(\xi_{3}\right)\left(\Phi_{1}\left(\xi_{1}\right) X_{n}+\left(\Phi_{1}\left(\xi_{1}\right)-I\right) A_{1}^{-1} B_{1} U\right)+\left(\Phi_{3}\left(\xi_{3}\right)-I\right) A_{3}^{-1} B_{3} U\right) \\
d<0.5: \quad X_{n+1} & =F\left(X_{n}, \xi_{1}, \xi_{2}, \xi_{4}\right)=f_{4, \xi_{4}} \circ f_{2, \xi_{2}} \circ f_{1, \xi_{1}}\left(X_{n}\right) \\
& =\Phi_{4}\left(\xi_{4}\right)\left(\Phi_{2}\left(\xi_{2}\right)\left(\Phi_{1}\left(\xi_{1}\right) X_{n}+\left(\Phi_{1}\left(\xi_{1}\right)-I\right) A_{1}^{-1} B_{1} U\right)\right)
\end{aligned}
$$

where $f \circ g(t)=f(g(t))$.

Since the closed loop control of voltage is used in the Buck converter, the switching function $S\left(X_{n}, d_{n}\right)$ can be derived as:

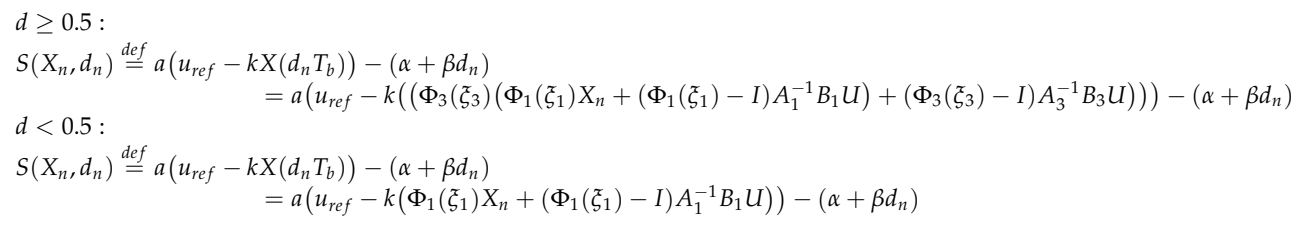


where $X_{n}$ is the initial state of period $n(n \in \mathrm{N}) ; k$ is voltage divider ratio; $a$ is the gain of the error amplifier; vector $P=[0100]$ is a selection matrix of the Buck converter output voltage, $\alpha=U_{L}$, $\beta=U_{H}-U_{L}$. The on and off state of the switch $S$ should change at the moment $S\left(X_{n}, d_{n}\right)=0$.

As can be seen from Equation (10), when the initial state of period $n$ is determined, the duty cycle of the mapping period can be derived, and then according to Equation (9), the discrete mapping of the system can be obtained.

As mentioned before, the maximum Jacobi matrix eigenvalue is used to study the stability of the system at period-one steady state. The states repeat periodically when the system operates in period-one [32], i.e., $X_{n+1}=X_{n}$. So the periodic fixed point can be derived as:

$$
\begin{aligned}
& d \geq 0.5: \\
& x^{*}=\left(I-\Phi_{4}\left(\frac{T_{b}}{2}\right) \Phi_{1}\left(\frac{T_{b}}{2}\right)\right)^{-1} \Phi_{4}\left(T_{b}-d_{n} T_{b}\right)\left(\Phi_{3}\left(\frac{2 d_{n} T_{b}-T_{b}}{2}\right)\left(\Phi_{1}\left(\frac{T_{b}}{2}\right)-I\right) A_{1}^{-1} B_{1} E+\left(\Phi_{3}\left(\frac{2 d_{n} T_{b}-T_{b}}{2}\right)-I\right) A_{3}{ }^{-1} B_{3} E\right) \\
& d<0.5: \\
& x^{*}=\left(I-\Phi_{4}\left(\frac{T_{b}}{2}\right) \Phi_{1}\left(\frac{T_{b}}{2}\right)\right)^{-1} \Phi_{4}\left(\frac{T_{b}}{2}\right)\left(\Phi_{2}\left(\frac{T_{b}}{2}-d_{n} T_{b}\right)\left(\Phi_{1}\left(d_{n} T_{b}\right)-I\right)\right) A_{1}{ }^{-1} B_{1} E
\end{aligned}
$$

According to Equations (9) and (10), the Jacobi matrix can be derived, as Equation (12) shows, when $d$ is larger than 0.5 [35]:

$$
J\left(X_{n}\right)=\frac{\partial F}{\partial X_{n}}-\frac{\partial F}{\partial d_{n}}\left(\frac{\partial S}{\partial d_{n}}\right)^{-1}\left(\frac{\partial S}{\partial X_{n}}\right)
$$

where $\frac{\partial F}{\partial X_{n}}=\Phi_{4}\left(\frac{T_{b}}{2}\right) \Phi_{1}\left(\frac{T_{b}}{2}\right), \frac{\partial F}{\partial d_{n}}=T_{b} \Phi_{4}\left(T_{b}-d_{n} T_{b}\right) B_{3} E, \frac{\partial S}{\partial X_{n}}=-a k P \Phi_{3}\left(\frac{2 d_{n} T_{b}-T_{b}}{2}\right) \Phi_{1}\left(\frac{T_{b}}{2}\right)$,

$$
\frac{\partial S}{\partial d_{n}}=-a k \mathrm{P}\left(A_{3} T_{b} \Phi_{3}\left(\frac{2 d_{n} T_{b}-T_{b}}{2}\right)\left(\Phi_{1}\left(\frac{T_{b}}{2}\right) X_{n}+\left(\Phi_{1}\left(\frac{T_{b}}{2}\right)-I\right) A_{1}{ }^{-1} B_{1} E\right)+T_{b} \Phi_{3}\left(\frac{2 d_{n} T_{b}-T_{b}}{2}\right) B_{3} E\right)-\beta
$$

When $d$ is smaller than 0.5 , the Jacobi matrix can be derived as:

$$
J\left(X_{n}\right)=\frac{\partial F}{\partial X_{n}}-\frac{\partial F}{\partial d_{n}}\left(\frac{\partial S}{\partial d_{n}}\right)^{-1}\left(\frac{\partial S}{\partial X_{n}}\right)
$$

where $\frac{\partial F}{\partial X_{n}}=\Phi_{4}\left(\frac{T_{b}}{2}\right) \Phi_{1}\left(\frac{T_{b}}{2}\right), \frac{\partial F}{\partial d_{n}}=T_{b} \Phi_{1}\left(\frac{T_{b}}{2}-d_{n} T_{b}\right) B_{1} E, \frac{\partial S}{\partial X_{n}}=-a k P \Phi_{1}\left(d_{n} T_{b}\right)$,

$$
\frac{\partial S}{\partial d_{n}}=-a k \mathrm{P}\left(A_{1} T_{b} \Phi_{1}\left(d_{n} T_{b}\right) X_{n}+T_{b} \Phi_{1}\left(d_{n} T_{b}\right) B_{1} E\right)-\beta
$$

Substituting Equations (11)-(13), the maximum Jacobi matrix eigenvalues can be obtained at the periodic fixed point, from which the stability of the system can be derived.

\section{Bifurcation Studies of Buck-Inverter and Maximum Jacobi Matrix Eigenvalue Verification}

In this section, bifurcation diagrams of the Buck-inverter will be presented and verified by the Jacobi matrix eigenvalue. The parameters of the system are shown in Table 1.

Table 1. Parameters of the system.

\begin{tabular}{cccc}
\hline Parameter & Value & Parameter & Value \\
\hline$L$ & $99.39 \mu \mathrm{H}$ & $a$ & 1 \\
$C$ & $48.6 \mu \mathrm{F}$ & $L_{p}$ & $327.84 \mu \mathrm{H}$ \\
$R_{L}$ & $0.7 \Omega$ & $C_{p}$ & $193.16 \mathrm{nF}$ \\
$f_{b}$ & $20 \mathrm{kHz}$ & $R_{p}$ & $2.19 \Omega$ \\
$f_{i}$ & $20 \mathrm{kHz}$ & $U_{L}$ & $1 \mathrm{~V}$ \\
$k$ & 1 & $U_{H}$ & $4 \mathrm{~V}$ \\
\hline
\end{tabular}

Bifurcation diagrams are a typical tool for analyzing nonlinear dynamic behaviors. Usually, it is obtained by stroboscopic sampling, and plotted between the input sensitive parameter in the 
$X$-axis and the behavior of a state space variable in the $Y$-axis [36]. Herein, the inductor current $i_{L}$ is taken as the state variable, while the reference voltage $u_{\text {ref }}$ and the input voltage $E$ are taken as the sensitive parameters.

In order to obtain the bifurcation diagram, a stroboscopic sampling algorithm was added to the time responses of the system to pick out the data at the beginning of each operating period $T_{b}\left(T_{b}=T_{i}\right)$ of $i_{L}$. For each set of sensitive parameter, 100 data points were sampled when the system was running in steady state. The initial states of each set of sensitive parameters are the steady state of the previous (For example, when we derived the time response when $u_{\text {ref }}=13 \mathrm{~V}$, the previous $u_{\text {ref }}$ is $12.9 \mathrm{~V}$. We just took the steady state (not zero vector) when $u_{r e f}=12.9 \mathrm{~V}$ as the initial state). The obtained bifurcation diagram is shown in Figure 4, where Figure $4 \mathrm{a}, \mathrm{c}$ take $u_{r e f}$ and $E$ as the sensitive parameters respectively (In Figure $4 \mathrm{a}, E$ is set to be $20 \mathrm{~V}$ while $u_{r e f}$ is set to be $12.5 \mathrm{~V}$ in Figure $4 \mathrm{c}$ ).

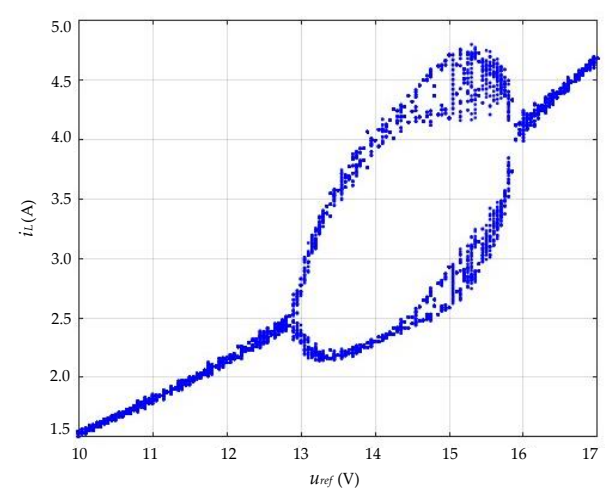

(a)

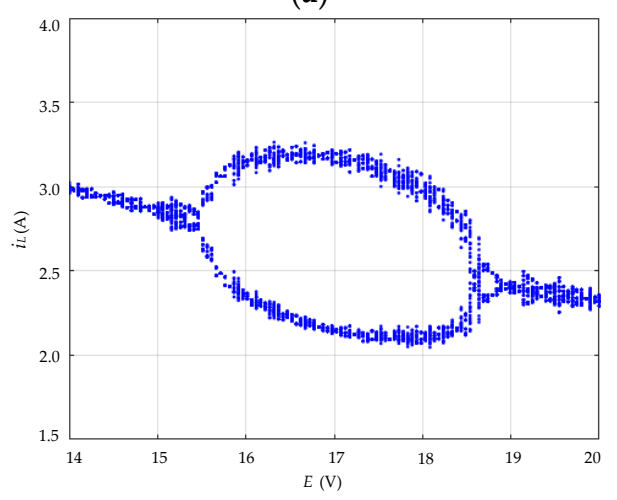

(c)

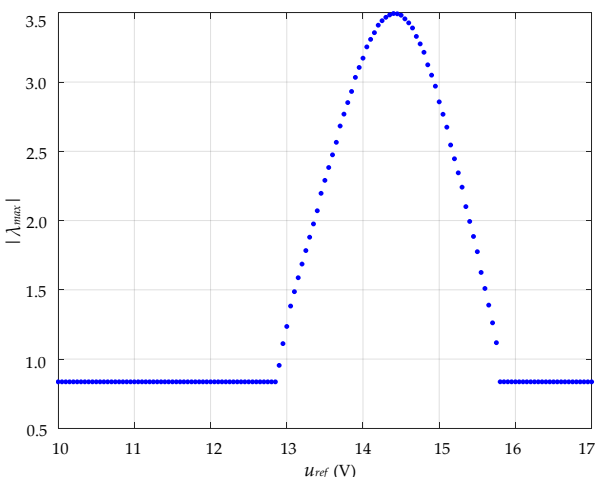

(b)

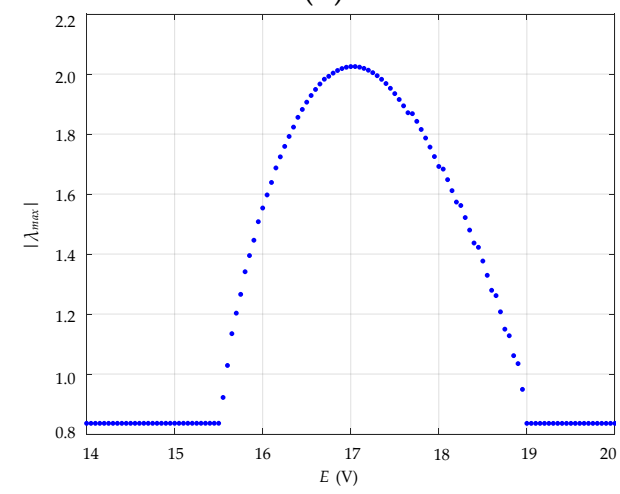

(d)

Figure 4. Bifurcation diagrams and maximum Jacobi matrix eigenvalues verification of the system with different sensitive parameters: (a) Bifurcation diagram with $u_{\text {ref }}$ as the sensitive parameter; (b) maximum Jacobi matrix eigenvalues with $u_{\text {ref }}$ as the sensitive parameter; (c) bifurcation diagram with $E$ as the sensitive parameter; (d) maximum Jacobi matrix eigenvalues with $E$ as the sensitive parameter.

As can be seen from Figure $4 \mathrm{a}$, as the reference voltage $u_{\text {ref }}$ increases, complex bifurcation occurs. The system exhibits period-one behavior for low values of $u_{\text {ref, }}$, which remains stable up to $u_{r e f} \approx 12.8 \mathrm{~V}$. Then it bifurcates into period-two operating mode. This dynamic lasts up to $u_{r e f} \approx 14.5 \mathrm{~V}$ and transits to period-four orbits from $u_{\text {ref }} \approx 14.5 \mathrm{~V}$ to $15.8 \mathrm{~V}$ except for a chaotic window at about $15.1 \mathrm{~V}$ to $15.8 \mathrm{~V}$. At $u_{\text {ref }} \approx 15.8 \mathrm{~V}$, the system goes back to period-one and lasts up to $17 \mathrm{~V}$. As for the bifurcation diagram shown in Figure $4 \mathrm{c}$, bifurcation occurs at about $E \approx 15.5 \mathrm{~V}$, and goes back to period-one at $E \approx 18.8 \mathrm{~V}$.

Figure $4 \mathrm{~b}$, $\mathrm{d}$ shows the maximum Jacobi matrix eigenvalue of the system with respect to the sensitive parameters. As can be seen from Figure $4 b, d$, the maximum Jacobi matrix eigenvalue only considers the stability of the periodic fixed point, and cannot determine whether the system is 
bifurcation or chaos when the absolute value is larger than 1. Comparing Figure $4 a-d$, the bifurcation diagrams correspond well to the maximum Jacobi matrix eigenvalues, and the unstable states (bifurcation of chaos) occur when the absolute value of the maximum Jacobi matrix eigenvalue is larger than 1 . So the bifurcation diagrams are verified by the maximum Jacobi matrix eigenvalue, and this accuracy is further verified by the results of the experiment.

\section{Cell Mapping Method and the Coexistence of Attractors of the Buck-Inverter}

Initial sensitivity and parameter sensitivity are two typical properties of nonlinear systems [36]. Above, the bifurcation diagrams in Figure 4a,c show the parameter sensitivity of the Buck-inverter that the system converges to different steady states (e.g., period-one, period-two or chaos) when $u_{\text {ref }}$ and $E$ varies. In this section, we will study the initial states sensitivity of the Buck-inverter, considering that the system starts from different initial states (as mentioned before, the bifurcation diagrams shown in Figure $4 a, c$ are derived by setting the steady state of the previous parameters as the initial states). References $[13,14,19]$ show that the Buck converter and inverter have coexistence of attractors, and that the attractors are different corresponding to different initial values. As for the Buck-inverter, there are four state variables. In order to find the attractors quickly, we here introduce an analytical method: the cell mapping method.

The cell mapping method can be used to find the domain of a nonlinear system [37]. Since there is no need to repeat the calculations when the cell enters into a known cell, the method can find the domain quickly. To help understand the cell mapping method, a two-dimension system is used to illustrate. Firstly, the cell can be defined as $C_{c}(i, p, s)$, where $c$ indicates the cell number, $i, p$ and $s$ indicate the index of the attractors, the period number and steps to enter the period, respectively.

The cell mapping process is shown in Figure 5, where $C_{5}, C_{6}$ and $C_{7}$ of red blocks indicate a period-three cycle with the step as zero, while $C_{4}$ (the green block) indicates a way to period-three with the step as one. $C_{1}$ (the blue block) indicates a period-one cycle with the step as zero. Considering the cell $C_{2}, C_{3}$ and $C_{8}$ of white blocks, $C_{3}$ and $C_{8}$ can also be the way to a period-three cycle, with the difference being that $C_{3}$ need two steps to get to the period-three cycle, while $C_{8}$ needs one step. $C_{2}$ is the way to period-one, and the step is one.

As mentioned above, cell mapping is a quick and effective way to search the domain of the attractors, assuming the colorful blocks $C_{1}, C_{4}, C_{5}, C_{6}, C_{7}$ are known cells, so $C_{2}, C_{3}$ and $C_{8}$ need only one step to derive the overall trajectory. For example, $C_{3}$ mapping to $C_{4}$ needs one step, so we can derive that $C_{3}$ belong to period-three domain, since $C_{4}, C_{5}, C_{6}$ and $C_{7}$ all belong to the period-three domain, so we do not need to repeat the mapping, e.g., mapping from $C_{4}$ to $C_{5}$.

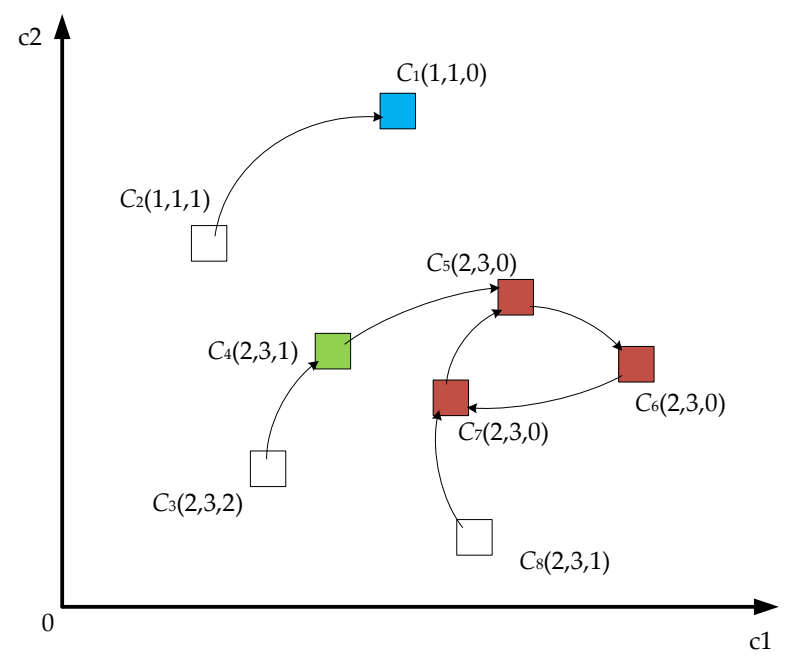

Figure 5. Cell mapping example of a two-dimension system. 
As the parameters of the Buck-inverter has been determined in Table 1, the possible attractors can also be quickly derived. Set $u_{r e f}=11,12.5$ and $16.6 \mathrm{~V}$ and $E=20 \mathrm{~V}$, the possible initial value ranges are derived from the simulation study, and this is also verified by the results of the experiment: $i_{L}=[0,8]$ $\mathrm{A} ; u_{c}=[0,20] \mathrm{V} ; i_{p}=[-10,10] \mathrm{A} ; u_{p}=[-300,300] \mathrm{V}$. Then we divide the coordinate axis $\left(i_{L}, u_{c}, i_{p}, u_{p}\right)$ into a countable number of intervals with uniform size (the uniform size may be different with respect to different axes), and assume the intervals belong to same domain of attractors. Here, we divide $i_{L}$, $u_{c}, i_{p}$ and $u_{p}$ into 8,20, 21 and 61 intervals, so that the entire cell number is 204,960, and then we can derive the possible attractors of the global domain, and derive the cell numbers of different attractors. The results are shown in Table 2, where the "Others" indicates that the period is beyond the maximum searching period (in this paper, the maximum searching period is set to be 10), or the steady state is chaos. It should be noted that the states of chaos are stochastic, so in the experimental verification section, we only cared about the period attractors.

As can be seen from Table 2, only period-one attractors exist in the system when $u_{\text {ref }}=11 \mathrm{~V}$, while the coexistence of period-one and period-three attractors happens in the system when $u_{r e f}=12.5 \mathrm{~V}$, and coexistence of period-one, period-three and period-four attractors appears in the system when $u_{r e f}=16.6 \mathrm{~V}$. These attractors are verified by the results of the experiment.

Table 2. Cell numbers and attractors of different $u_{\text {ref }}$.

\begin{tabular}{ccc}
\hline $\boldsymbol{u}_{\boldsymbol{r e f}}$ & Attractors & Cell Numbers \\
\hline $11 \mathrm{~V}$ & Period-one & 204,960 \\
\hline \multirow{3}{*}{$12.5 \mathrm{~V}$} & Period-one & 116,473 \\
& Period-three & 70,974 \\
& Others & 17,513 \\
\hline \multirow{3}{*}{$16.6 \mathrm{~V}$} & Period-one & 102,467 \\
& Period-three & 47,625 \\
& Period-four & 35,219 \\
& Others & 19,649 \\
\hline
\end{tabular}

\section{Experimental Verification}

To verify the bifurcation diagrams and coexistence of attractors as shown above, an experiment prototype was built with the measured main parameters shown in Table 1.

With the input disturbance (which is a change of the initial values by disturbance), the coexistence of attractors can be found. Normally, if the system has only one steady state, the different initial values will definitely lead the system to the same steady state. However, if the system has a coexistence of attractors, then the steady states are not the same.

Three points mentioned in Section 4 of $u_{\text {ref }}$, i.e., $11 \mathrm{~V}, 12.5 \mathrm{~V}$ and $16.6 \mathrm{~V}$, have been tested. The experiment results are shown in Figure 6a-f respectively. Where channel 1 is the inductor current $i_{L}$. channel 2 is the control signal of switch $S 1$. Channel 3 is the reference voltage $u_{\text {ref. }}$.

It can be seen from Figure 6 that the coexistence of attractors really exists in the Buck-inverter. Figure $6 \mathrm{~b}, \mathrm{c}$ show that when $u_{\text {ref }}=12.5 \mathrm{~V}$, the system may result in period-one orbit or period-three orbit with different initial values. Figure $6 \mathrm{~d}-\mathrm{f}$ show that when $u_{r e f}=16.6 \mathrm{~V}$, the system may result in period-one orbit or period-three orbit or period-four orbit with different initial values. As for $u_{r e f}=11 \mathrm{~V}$, only period-one attractors in the system are shown in Figure 6a. Compared with the theoretical results shown in Figure 4 and Table 2, it can be seen that the bifurcation characteristics and coexistence of attractors match very well. So the bifurcation diagrams and coexistence of attractors are verified. 


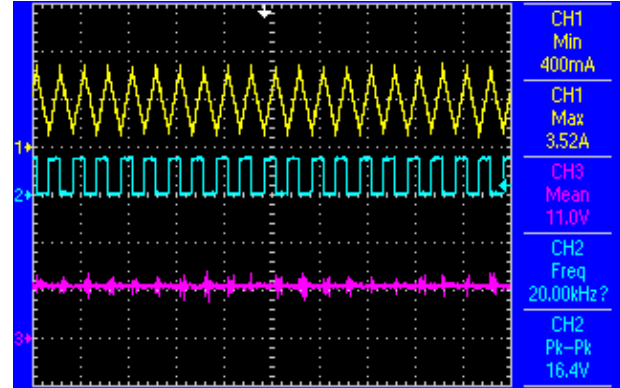

(a)

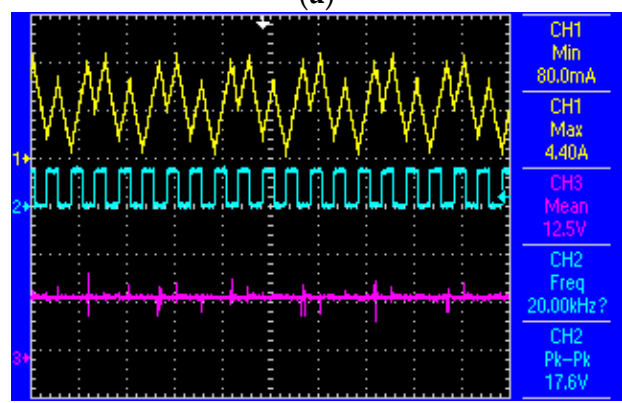

(c)

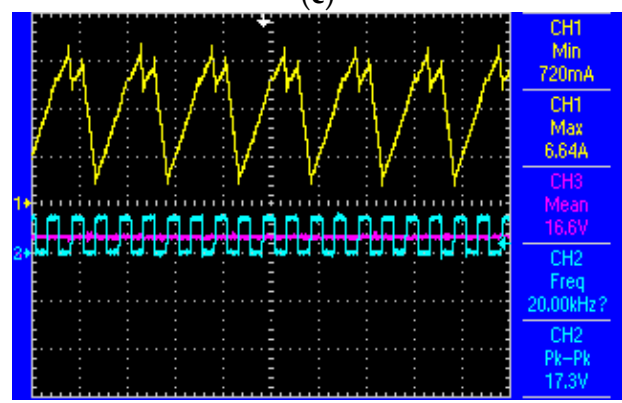

(e)

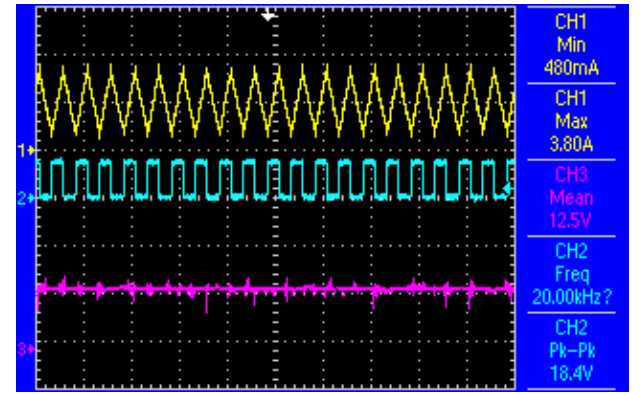

(b)

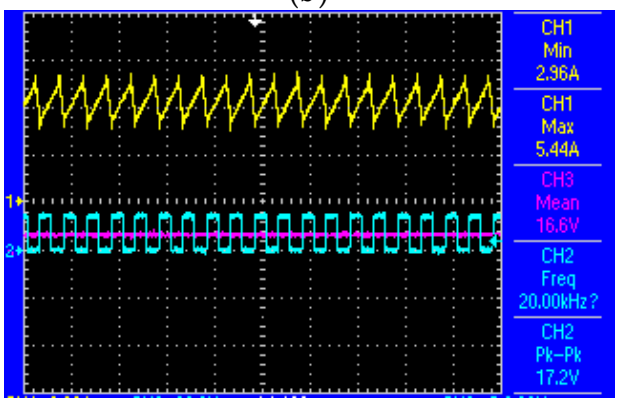

(d)

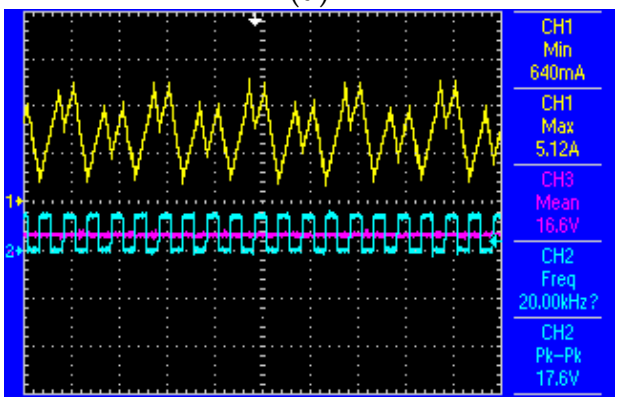

(f)

Figure 6. Experiment results of the coexistence of attractors phenomena: (a) $u_{\text {ref }}=11 \mathrm{~V}$ with period-one orbit; (b) $u_{\text {ref }}=12.5 \mathrm{~V}$ with period-one orbit; (c) $u_{\text {ref }}=12.5 \mathrm{~V}$ with period-three orbit; (d) $u_{\text {ref }}=16.6 \mathrm{~V}$ with period-one orbit; (e) $u_{r e f}=16.6 \mathrm{~V}$ with period-three orbit; (f) $u_{r e f}=16.6 \mathrm{~V}$ with period-four orbit.

\section{Conclusions}

This paper studies the bifurcation phenomena of a voltage-controlled Buck-inverter cascade system. A state-flow chart has been introduced to illustrate the complex relations among the linear operating modes, on the basis of which the time responses of the system have been derived. The bifurcation diagrams have been obtained with respect to the reference voltage and the input voltage, and verified by the maximum Jacobi matrix eigenvalues. Specifically, the coexistence of attractors has been revealed and investigated globally utilizing the cell mapping method of analysis. Both the bifurcation diagrams and the coexistence of attractors have been verified by the experiment results.

It can be concluded that in a cascade power electronics system, complex nonlinear behaviors may exist. When we design such systems, the method presented in this paper can be applied to learn their bifurcation characteristics in the full range of variations of the key parameters such as load, input voltage and reference voltage, etc. Then boundaries of operation can be defined by checking the system in the bifurcation zone as to whether the performance requirements can be satisfied or not. Parameter optimization or specific control strategies dealing with the nonlinear phenomena should be further investigated and implemented if the system performance cannot meet the requirements.

Acknowledgments: This research is supported by National Natural Science Foundation of China (61573074, 51477020), and the Fundamental Research Funds for the Central Universities (106112015CDJXY17002). 
Author Contributions: Chunsen Tang and Xin Dai conceived and designed the experiments; Xiaofei Li performed the experiments; Xiaofei Li, Chunsen Tang and Xin Dai wrote the paper. Aiguo Patrick Hu and Sing Kiong Nguang analyzed the experiment phenomenon and revised the paper; All authors have contributed to the editing and proofreading of this paper.

Conflicts of Interest: The authors declare no conflict of interest.

\section{References}

1. Geng, Y.; Li, B.; Yang, Z.; Lin, F.; Sun, H. A high efficiency charging strategy for a supercapacitor using a wireless power transfer system based on Inductor/Capacitor/Capacitor (LCC) compensation topology. Energies 2017, 10, 135. [CrossRef]

2. Pevere, A.; Petrella, R.; Mi, C.C.; Zhou, S.J. Design of a high efficiency $22 \mathrm{~kW}$ wireless power transfer system for EVs fast contactless charging stations. In Proceedings of the IEEE International Electric Vehicle Conference (IEVC), Florence, Italy, 17-19 December 2014; pp. 1-7.

3. Zerouali, S.; Allag, A.; Minoune, S.M.; Hamida, A.H. An adaptive control applied to series resonant inverter for induction heating. In Proceedings of the IEEE International Electric Vehicle Conference (IEVC), Newcastle upon Tyne, UK, 6-8 September 2008; pp. 633-636.

4. Carretero, C.; Lucia, O.; Acero, J.; Burdio, J.M. Phase-shift control of dual half-bridge inverter feeding coupled loads for induction heating purposes. Electron. Lett. 2011, 47, 670-671. [CrossRef]

5. Zadeh, M.K.; Ghoachani, R.G.; Martin, J.P.; Pierfederici, S.; Mobarakeh, B.N.; Molinas, M. Discrete-Time tool for stability analysis of dc power electronics-based cascaded systems. IEEE Trans. Power Electron. 2016, 32, 652-667. [CrossRef]

6. Hamill, D.C.; Deane, J.H. B.; Jefferies, D.J. Modeling of chaotic dc-dc converters by iterated nonlinear mappings. IEEE Trans. Power Electron. 1992, 7, 25-36. [CrossRef]

7. Flegar, I.; Pelin, D.; Zazeck, D. Bifurcation diagrams of the buck converter. In Proceedings of the International Conference on Electronics, Circuits and Systems, Los Angeles, CA, USA, 4-6 September 2002; pp. $975-978$.

8. Li, W.; Zhang, J. Research of period doubling bifurcation in pwm buck dc-dc converter using harmonic balance. In Proceedings of the International Colloquium on Computing, Communication, Control, and Management, Guangzhou, China, 4-5 August 2008; pp. 281-284.

9. Chan, W.C.Y.; Tse, C.K. Study of bifurcations in current-programmed dc/dc boost converters: From quasiperiodicity to period-doubling. IEEE Trans. Circuits Syst. I 1997, 44, 1129-1142. [CrossRef]

10. Cheng, K.W.E.; Liu, M.; Wu, J. Chaos study and parameter-space analysis of the dc-dc buck-boost converter. IEEE Proc. Electron. Power Appl. 2003, 150, 126-138. [CrossRef]

11. Debbat, M.B.; Aroudi, A.E.; Giral, R.; Martinez, S.L. Stability analysis and bifurcations of SEPIC dc-dc converter using a discrete-time model. In Proceedings of the IEEE International Conference on Industrial Technology, Bangkok, Thailand, 11-14 December 2002; pp. 1055-1060.

12. Iqbal, S.; Zang, X.; Zhu, Y.; Zhao, J. Study of bifurcation and chaos in dc-dc boost converter using discrete-time map. In Proceedings of the International Conference on Mechatronics and Control, Jinzhou, China, 3-5 July 2014; pp. 1813-1817.

13. Banerjee, S. Coexisting attractors, chaotic saddles, and fractal basins in a power electronic circuit. IEEE Trans. Circuits Syst. I 1997, 44, 847-849. [CrossRef]

14. Morcillo, J.; Olivar, G. Coexistence of solutions in a dc-dc buck converter controlled by sine wave. In Proceedings of the IEEE IX Latin American and IEEE Colombian Conference on Automatic Control and Industry Applications, Bogota, Colombia, 1-4 October 2011; pp. 1-6.

15. Tse, C.K. Flip bifurcation and chaos in three-state boost switching regulators. IEEE Trans. Circuits Syst. I 1994, 41, 16-23. [CrossRef]

16. Lu, H.H.C.; Zhou, Y.; Chi, K.T. Fast-scale instability in a PFC boost converter under average current-mode control. Int. J. Circuit Theory Appl. 2013, 31, 611-624.

17. Robert, B.; Robert, C. Border collision bifurcations in a one-dimensional piecewise smooth map for a PWM current-programmed H-bridge inverter. Int. J. Control 2012, 75, 1356-1367. [CrossRef]

18. Shankar, D.P.; Govindarajan, U.; Karunakaran, K. Period-bubbling and mode-locking instabilities in a full-bridge DC-AC buck inverter. IET Power Electron. 2013, 6, 1956-1970. [CrossRef] 
19. Okafor, N.; Zahawi, B.; Giaouris, D.; Banerjee, S. Chaos, coexisting attractors, and fractal basin boundaries in dc drives with full-bridge converter. In Proceedings of the IEEE International Symposium on Circuits and Systems, Paris, France, 30 May-2 June 2010; pp. 129-132.

20. Wang, L.; Zhou, Y.; Chen, J. Study of nonlinear behaviour and chaotic control in parallel-connection buck converters. In Proceedings of the International Conference on Electrical and Control Engineering, Yichang, China, 10-12 June 2011; pp. 5003-5006.

21. Lu, H.H.C.; Tse, C.K. Bifurcation behavior in parallel-connected buck converters. IEEE Trans. Circuits Syst. I 2001, 48, 233-240.

22. Lu, H.H.C.; Tse, C.K. Study of low-frequency bifurcation phenomena of a parallel-connected boost converter system via simple averaged models. IEEE Trans. Circuits Syst. I 2003, 50, 679-685. [CrossRef]

23. Mandal, K.; Banerjee, S.; Chakraborty, C. Symmetry-Breaking bifurcation in series-parallel load resonant dc-dc converters. IEEE Trans. Circuits Syst. I 2003, 60, 778-787. [CrossRef]

24. Mandal, K.; Banerjee, S.; Chakraborty, C. Symmetry-Breaking bifurcation in load resonant dc-dc converters. In Proceedings of the IEEE International Symposium of Circuits and Systems, Rio, Brazil, 15-18 May 2011; pp. 1327-1330.

25. Hu, L.; Liu, Y.; Teng, W.; Zhou, C. Nonlinear coupled dynamics of a rod fastening rotor under rub-impact and initial permanent deflection. Energies 2016, 9, 883. [CrossRef]

26. Xie, F.; Zhang, B.; Qiu, D.; Jiang, Y. Non-linear dynamic behaviours of DC cascaded converters system with multi-load converters. IET Power Electron. 2016, 9, 1093-1102. [CrossRef]

27. Mandal, K.; Banerjee, S.; Chakraborty, C.; Chakraborty, M. Bifurcations in frequency controlled load resonant dc-dc converters. In Proceedings of the IEEE International Symposium on Circuits and Systems, Seoul, Korea, 20-23 May 2012; pp. 1135-1138.

28. Sun, Y.; Zhang, H.; Hu, A.P.; Tang, C.S.; Xiang, L.J. The recognition and control of nonideal soft-switching frequency for wireless power transfer system based on waveform identification. IEEE Trans. Power Electron. 2016, 32, 6617-6627. [CrossRef]

29. Wu, J.; Zhao, C.; Lin, Z.; Du, J.; Hu, Y.; He, X. Wireless power and data transfer via a common inductive link using frequency division multiplexing. IEEE Trans. Ind. Electron. 2015, 62, 7810-7820. [CrossRef]

30. Bertoni, N.; Frattini, G.; Massolini, R.G.; Pareschi, F.; Rovatti, R.; Setti, G. An analytical approach for the design of Class-E resonant dc-dc converters. IEEE Trans. Power Electron. 2016, 31, 7701-7713. [CrossRef]

31. Chen, H.; Fu, K.; Xu, H.; Ding, K. Implementation of hybrid control for Bi-directional dc-dc converter. In Proceedings of the IEEE Conference on Cyber Technology in Automation, Control and Intelligent Systems, Hong Kong, China, 4-7 June 2014; pp. 669-674.

32. Tang, C.S.; Sun, Y.; Su, Y.G.; Nguang, S.K.; Hu, A.P. Determining multiple steady-state zcs operating points of a switch-mode contactless power transfer system. IEEE Trans. Power Electron. 2009, 24, 416-425. [CrossRef]

33. Tang, C.S.; Dai, X.; Wang, Z.H.; Hu, A.P. Frequency bifurcation phenomenon study of a soft switched push-pull contactless power transfer system. In Proceedings of the IEEE Conference on Industrial Electronics and Applications, Beijing, China, 21-23 June 2011; pp. 1981-1986.

34. Li, M.; Dai, D.; Ma, X.; Iu, H.H.C. Fast-Scale period-doubling bifurcation in voltage-mode controlled full-bridge inverter. In Proceedings of the IEEE International Symposium on Circuits and Systems, Seattle, WA, USA, 18-21 May 2008; pp. 2829-2832.

35. Alligood, K.T.; Sauer, T.D.; Yorke, J.A. Chaos: An Introduction to Dynamical Systems; Springer: New York, NY, USA, 1996.

36. Yesodha, V.; Kavipriya, R.; Jooshna, T.S.; Rajini, V. Analysis of chaos and bifurcation in dc-dc converter using Matlab. In Proceedings of the International Conference on Circuits, Power and Computing, Nāgercoil, India, 21-22 March 2013; pp. 481-487.

37. Li, B.; Yang, R.; Yu, L. Application of cell mapping method in chaotic detection of asynchronous motor at low speed. In Proceedings of the European Conference on Power Electronics and Applications, Karlsruhe, Germany, 5-9 September 2016; pp. 1-10.

(C) 2017 by the authors. Licensee MDPI, Basel, Switzerland. This article is an open access article distributed under the terms and conditions of the Creative Commons Attribution (CC BY) license (http://creativecommons.org/licenses/by/4.0/). 